\title{
ArcheoSciences
}

Revue d'archéométrie

\section{Approche analytique pour l'étude des ivoires archéologiques. Les défenses d'éléphant du site de Jinsha (1200-650 BC, Sichuan, Chine)}

Analytical Approach for the Study of Archaeological Ivory Elephant Tusks from the Site of Jinsha (1200-650 BC, Sichuan, China)

Dounia Large, Katharina Müller et Ina Reiche

\section{(2) OpenEdition}

Journals

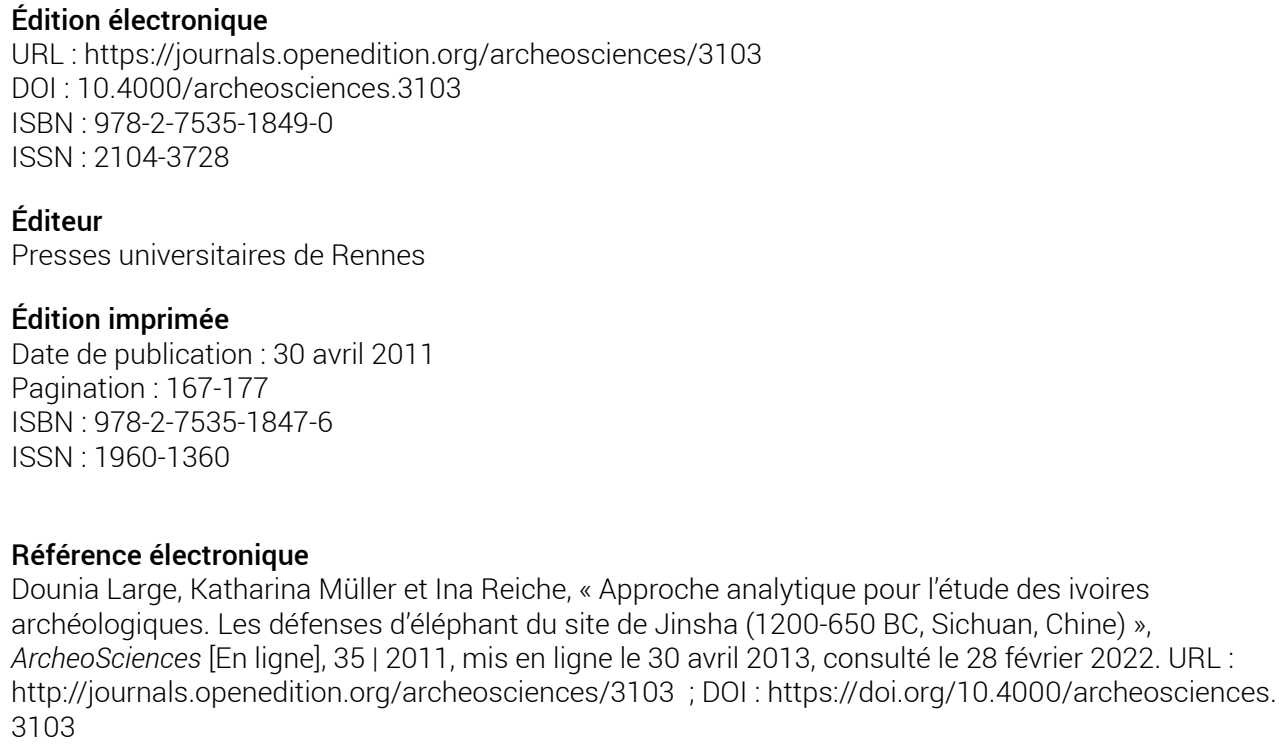




\title{
Approche analytique pour l'étude des ivoires archéologiques. Les défenses d'éléphant du site de Jinsha (1200-650 BC, Sichuan, Chine)
}

\author{
Analytical Approach for the Study of Archaeological Ivory \\ Elephant Tusks from the Site of Jinsha (1200-650 BC, Sichuan, China)
}

\author{
Dounia LARge*, **, Katharina MüLler* et Ina ReICHE*
}

\begin{abstract}
Résumé : Une méthode, plurianalytique et multiéchelle, a été développée et adaptée pour déterminer précisément l'état de conservation des ivoires d'éléphant archéologiques. Elle a pour but de caractériser non seulement la phase minérale de la dentine par des analyses élémentaires et spectrométriques, mais aussi la phase organique. De plus, le recours à l'imagerie électronique permet de décrire l'arrangement et les modifications de ces deux phases à micro- et nanoéchelle. Cette démarche a tout d'abord été appliquée extensivement à l'ivoire moderne d'éléphant afin d'établir une base de référence pour évaluer l'état de conservation des échantillons archéologiques. Du matériel provenant du site archéologique chinois de Jinsha présentant une forte fragilité a ensuite été analysé. La différence d'état de conservation entre les ivoires archéologiques et la référence moderne étudiée d'une part et les échantillons archéologiques entre eux d'autre part a pu être évaluée, confirmant l'efficacité de la méthode. Ces recherches montrent l'importance de l'étape de séchage de l'ivoire à sa sortie de sol lors des fouilles, en particulier pour des sites très humides comme celui de Jinsha.
\end{abstract}

Abtract: A multiscale analytical approach combining different complementary methods was developed and adapted to accurately determine the preservation state of archeological elephant ivories. It aimed not only at characterizing the mineral phase by elementary and spectrometric techniques but also the organic phase. Furthermore, the imbrications and the evolution of the two phases during burial was investigated by electron microcopy. This method is first applied to modern elephant ivory used as a reference for evaluating the preservation state of the archaeological samples. It is then used on a panel of fragile samples from the Chinese archeological site of Jinsha. The results clearly allow the evaluation of preservation state differences between modern and archeological samples. In addition, this analytical approach allows to discriminate between archeological samples. Furthermore, the results of this study highlight the importance of controlling the drying step of excavated humid ivory objects.

Mots clé : altération, éléphant, ivoire, Jinsha, MEB, MET, microtomographie X, PIXE.

Keywords: alteration, elephant, ivory, Jinsha site, microCT, PIXE, SEM, TEM.

* Laboratoire du Centre de Recherche et de Restauration des Musées de France (LCR2MF UMR 171 CNRS), Palais du Louvre - 14 quai François
Mitterrand, 75001 Paris, France. (ina.reiche@culture.gouv.fr) (katharina.mueller@culture.gouv.fr)
** De la Préhistoire à l'Actuel: Culture, Environnement et Anthropologie (PACEA UMR 5199 CNRS), Université Bordeaux 1 - Bâtiment B 18, Avenue
des Facultés, 33405 Talence Cedex, France. (d.Large@pacea.u-bordeaux1.fr) 


\section{INTRODUCTION}

Le terme " ivoire " désigne ici le matériau de base, ou dentine, des défenses d'éléphant. Les défenses sont généralement de grande taille atteignant 2 à $3 \mathrm{~m}$ en longueur et un diamètre de l'ordre de 20 à $30 \mathrm{~cm}$, pour un poids allant jusqu'à $120 \mathrm{~kg}$. Elles sont de forme conique avec une cavité contenant la pulpe dentaire et prolongée par un canal nerveux jusqu'à la pointe de la défense. La partie de défense exposée est couverte d'un cément d'un centimètre environ. Le reste, et donc la majorité de la masse de la défense, est composé de dentine [1].

L'ivoire étant très sensible aux variations d'humidité et de température ainsi qu’à la lumière, il fait partie des matériaux les plus difficiles à conserver. Lors de fouilles archéologiques, il a très souvent tendance à se déliter, voire à éclater en petits morceaux lorsqu'il est mis au jour ce qui implique la perte de la forme initiale de l'objet exhumé. Cet important problème de conservation se retrouve par exemple dans le cas des défenses d'éléphant retrouvées en contexte archéologique sur le site de Jinsha (Sichuan, Chine, 700-800 BC) qui ont initié la réflexion présentée ici.

Peu de recherches systématiques ont été réalisées jusqu'ici afin d'évaluer précisément l'état de conservation et de prédire le comportement au séchage à la sortie du sol de l'ivoire, probablement à cause de la complexité de ce biomatériau composite [2]. Cependant, il est nécessaire de bien comprendre l'état de conservation de ce matériau avant de pouvoir proposer des stratégies de conservation incluant des étapes de séchage et de consolidation, par exemple par imprégnation de résine.

Nous présentons donc ici une approche multianalytique développée pour une évaluation fine de l'état de conservation de l'ivoire archéologique. Cette approche a tout d'abord été appliquée sur de l'ivoire moderne afin de disposer de références adéquates avant de servir à l'étude de quelques fragments prélevés sur des défenses d'éléphant issues du site archéologique de Jinsha.

\section{Le SITE ARCHÉOLOGIQUE DE JINSHA (Sichuan, Chine)}

Le site archéologique de Jinsha se trouve près de la ville de Chengdu dans la province du Sichuan en République populaire de Chine (fig. 1). Il fut découvert accidentellement en février 2001 lors du percement de nouveaux égouts. Les similarités des quelques objets mis au jour avec ceux du site de Sanxingdui tout proche, qui a permis de découvrir une civilisation inconnue de l'Âge du Bronze chinois, montrèrent rapi-

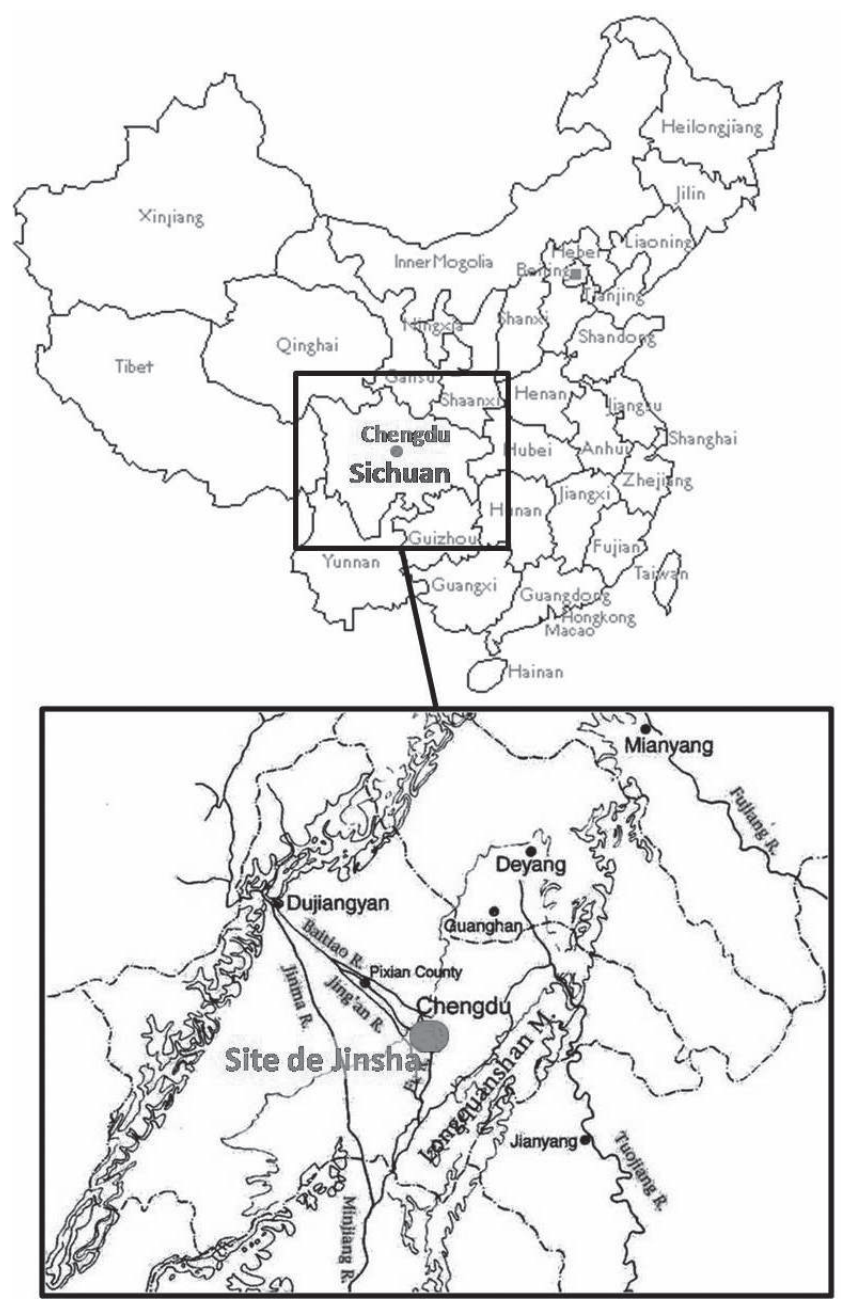

Figure 1: Localisation du site de Jinsha, à Chengdu, dans la province du Sichuan, République populaire de Chine.

Figure 1: Location of the Jinsha site, in the city of Chendu, in the Sichuan province, People's Republic of China.

dement l'intérêt du site et des fouilles furent programmées. Elles sont toujours actives aujourd'hui et ne cessent de s'étendre [3].

Le site est constitué de plusieurs centaines de structures bâties principalement dédiées au culte des ancêtres et des divinités datant du XII ${ }^{\mathrm{e}}$ au VI ${ }^{\mathrm{e}}$ siècle avant J.-C., et renfermant une grande quantité d'objets d'offrande et de cérémonie. Outre les sculptures monumentales en bronze, spécialité de cette civilisation, des pièces en feuille d'or, en jade, en pierre et en bois ont aussi été exhumées, ainsi que de très nombreuses défenses d'éléphant brutes (très probablement d'Asie), évaluées à plusieurs tonnes et apparemment d'utilisation rituelle. Cependant les archéologues se trouvent face à un grave problème de conservation de ces défenses : le sol est très humide, et malgré un climat lui-même humide, dès sa sortie l'ivoire a tendance à éclater et à se désagréger. 
Plusieurs stratégies pour préserver ces défenses ont été envisagées (traitement sur site, séchage contrôlé ou enrobage dans de la résine) sans pour autant trouver de solution satisfaisante. La direction des musées de Chengdu a alors sollicité une collaboration scientifique avec le laboratoire du C2RMF, ce qui a permis en 2007 d'obtenir des prélèvements d'ivoire représentatifs des différents états de conservation sur le site (tableau 1) : huit échantillons venant d'une défense traitée, probablement avec un biocide, une fois sèche (AI_ El_JI01a à h), deux venant d'une défense encore humide traitée probablement avec un biocide (AI_El_JI02a et b) et huit venant d'une défense humide non traitée (AI_El_JI03a à h). La nature exacte du traitement humide, pulvérisé sur site en surface de l'ivoire, ne nous est malheureusement pas connue.

Les échantillons d'ivoire moderne servant de référence pour déterminer l'état de conservation proviennent d'une défense d'éléphant d'Afrique adulte, issue de saisies douanières.

\section{Constitution de l'ivoire}

La dentine est un biomatériau nanocomposite, acellulaire, très organisé et poreux qui ne se renouvelle pas une fois formé mais qui continue deà croître durant la vie de l'animal $[4,5]$. La substance fondamentale de la dentine est similaire à celle de l'os et comporte deux phases, organique et miné- rale. La matrice organique, protéinique, est majoritairement constituée de fibres de collagène de type I dans lesquelles sont imprégnés des cristaux d'apatite carbonatée. L'ivoire se différencie des autres matériaux osseux de par sa structure microscopique compacte et plus homogène, par sa proportion légèrement plus élevée en phase organique mais aussi par une teneur relativement élevée en magnésiumMg [6]. Il est donc à la fois plus résistant et plus souple, ce qui en fait une matière idéale pour la sculpture.

À l'échelle macroscopique, l'ivoire d'éléphant montre un faciès caractéristique de lignes foncées, courbes et entrecroisées, appelé " réseau de Schreger ", que l'on reconnait aisément sur les sections transverses. À l'échelle microscopique, des tubules d'un diamètre de l'ordre d'un ou deux microns, et dont l'orientation varie, traversent la dentine dans toute son épaisseur. Ces tubules sont entourées de dentine péritubulaire, plus minéralisée que la dentine intertubulaire. Les tubules sont perpendiculaires aux faisceaux formés par les fibres de collagène. À l'échelle nanoscopique, l'organisation des fibrilles de collagène et des cristallites d'apatite est décrite en détail dans les références suivantes [1, 4, 7] (fig. 2).

\section{MéTHOdes et CONDitions EXPÉRIMENTALES}

Pour obtenir une bonne appréciation de l'état de conservation de l'ivoire, vue la complexité de ce biomatériau nanocomposite, il est nécessaire de caractériser à la fois la phase

\begin{tabular}{|c|c|c|c|c|}
\hline Défense & Prélèvements & Description & Unité stratigraphique & Vue globale \\
\hline AI_El_JI01 & $\begin{array}{c}\text { 8 prélèvements } \\
\text { JI01a à h }\end{array}$ & $\begin{array}{c}\text { Défense traité une fois } \\
\text { sèche }\end{array}$ & $\begin{array}{c}\text { T8204, environ couche } \\
15 \mathrm{~b}\end{array}$ \\
\hline AI_El_JI02 & $\begin{array}{c}\text { 2 prélèvements } \\
\text { J102a et b }\end{array}$ & $\begin{array}{c}\text { Défense traitée encore } \\
\text { humide }\end{array}$ & $\begin{array}{c}\text { T8204, environ couche } \\
15 \mathrm{~b}\end{array}$ \\
\hline AI_El_JI03 & $\begin{array}{c}\text { 8 prélèvements } \\
\text { JI03a à h }\end{array}$ & Défense non traitée & T8007 \\
\hline
\end{tabular}

Tableau 1 : Nomenclature et description des échantillons d'ivoire du site archéologique de Jinsha analysés dans cette étude. Table 1: Ivory samples from the archaeological site of Jinsha analyzed in this study: Inventory and description. 


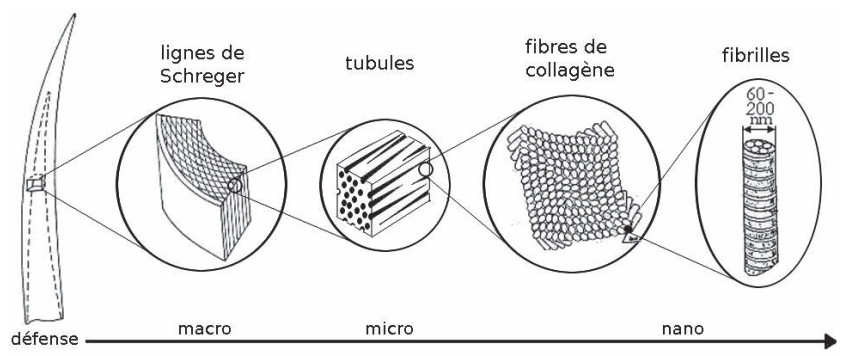

Figure 2 : Schéma de la constitution d'une défense d'ivoire allant du dm au nm. Pour des raisons de lisibilité, les particules minérales insérées dans la trame organique ne sont pas représentées. Plus de détails sur l'ultrastructure de l'ivoire peuvent être trouvés dans [4]. Figure 2: Schematic description of an elephant tusk constitution, from dm to $\mathrm{nm}$. For better clarity, the mineral particles embedded in the organic matrix are not been depected. For more details on ivory ultrastructure, please refer to [4].

minérale, la phase organique et leur imbrication, et ce de la macro à la nanoéchelle. Ceci nécessite l'utilisation de techniques d'analyse complémentaires (fig. 3).

\section{Microscopie électronique à balayage (MEB)}

Les observations au microscope électronique à balayage (MEB) ont été effectuées avec un Phillips XL 30CP. Afin d'assurer un bon état de surface et une bonne conduction des électrons, les prélèvements analysés ont été préalablement inclus dans de la résine, coupés et polis avant d'être recouverts d'une fine couche de carbone. L'observation s'est faite en mode d'électrons rétrodiffusés (BSE) avec une tension de $20 \mathrm{keV}$ pour un courant d'une centaine de $\mu \mathrm{A}$ pour l'ivoire moderne, et une tension de $15 \mathrm{keV}$ pour un courant d'environ $70 \mu \mathrm{A}$ pour les échantillons archéologiques.

\section{Micro-tomographie X avec une source synchrotron (SR-microCT)}

Les expériences de micro-tomographie par rayonnement synchrotron (SR-microCT) en mode de contraste d'absorption ont été réalisées sur la BAMline du synchrotron BESSY II, HZB à Berlin. Le rayonnement synchrotron est utilisé sous forme de rayons $\mathrm{X}$ monochromatiques $(14 \mathrm{keV})$ pour effectuer des tomographies à l'échelle du micron sur l'échantillon. Le porte-échantillon est mis en rotation avec 1440 prises de vue sur $180^{\circ}$. Le temps d'exposition peut varier de deux à cinq secondes par prise de vue (tableau 2) ce qui fait une durée totale d'analyse comprise entre deux et cinq heures [8].

L’ensemble des clichés est ensuite reconstruit informatiquement afin de pouvoir observer des tranches ou des volumes selon toutes les directions. Avec le logiciel VG Studio Max $2^{\circ}$, il est possible de sélectionner un volume d'intérêt et de choisir les densités correspondant au vide et au matériau. Ceci permet d'estimer la porosité globale de l'ivoire ainsi que de visualiser sa répartition en trois dimensions.

\section{Microscopie électronique à transmission (MET)}

Pour les observations au Microscope Electronique à Transmission (MET), des coupes ultrafines de 50 à $70 \mathrm{~nm}$ des échantillons préalablement imprégnés de polyméthyleméthacrylate (PMMA) ont été réalisées sur un Ultramicrotome Reichert Ultracut ${ }^{\oplus}$ au Centre Commun de Microscopie Electronique (CCME), Orsay avant d'être déposées sur des grilles de cuivre 200 mesh recouvertes d'une membrane de Carbon Formwar. Les observations ont ensuite été réalisées grâce au MET Philips EM208 «Darwin » à 80 kV au CCME, Orsay.

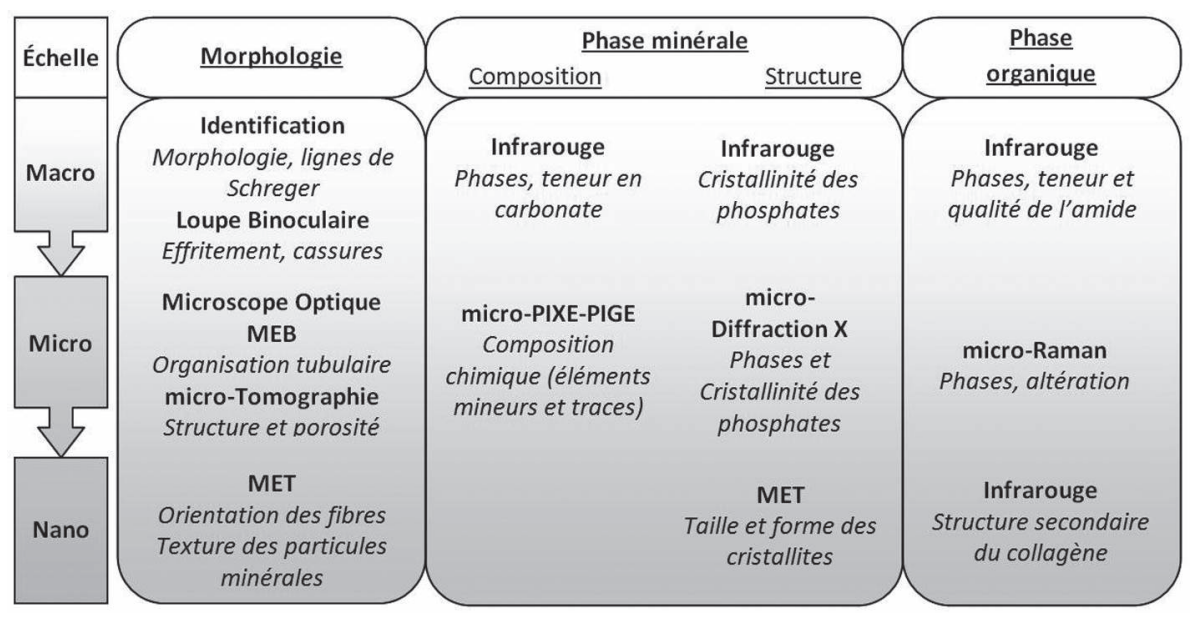

Figure 3 : Schéma méthodologique pour l'analyse des ivoires archéologiques.

Figure 3: Methodological chart for archaeological ivories characterization. 


\begin{tabular}{|c|c|c|}
\hline Échantillon & $\begin{array}{c}\text { Intensité de } \\
\text { l'anneau (mA) }\end{array}$ & $\begin{array}{c}\text { Temps } \\
\text { d'exposition (s) }\end{array}$ \\
\hline $\begin{array}{c}\text { Ivoire moderne } \\
\text { MI_El_1 }\end{array}$ & 218 & 3,0 \\
\hline AI_El_JI01d_ext & 288 & 2,0 \\
\hline AI_El_JI02b & 242 & 2,4 \\
\hline
\end{tabular}

Tableau 2 : Conditions expérimentales de micro-tomographie (SR-microCT) sur l'ivoire moderne et les ivoires archéologiques du site Jinsha, Chine.

Table 2: SR-microCT experimental conditions for modern ivory and archaeological samples from Jinsha site, China.

\section{Analyses élémentaires par émission de rayons $\mathrm{X}$ induite par un microfaisceau de protons (microPIXE)}

Les analyses par émission de rayons $\mathrm{X}$ induite par proton (microPIXE) ont été réalisées grâce à la ligne du microfaisceau extrait de l'Accélérateur Grand Louvre d'Analyse Elémentaire (AGLAE) avec des protons de $3 \mathrm{MeV}$, un courant de $1 \mathrm{nA}$ et un diamètre de faisceau de $50 \mu \mathrm{m}$. Les mesures sont effectuées grâce à deux détecteurs de rayons $\mathrm{X}$, basse $(1 \mathrm{à} 10 \mathrm{keV})$ et haute ( 4 à $20 \mathrm{keV}$ ) énergie. Les spectres de haute et de basse énergies sont ensuite dépouillés grâce aux logiciels GUPIX et TRAUPIX [9].

\section{Spectroscopie infrarouge à transformée de Fourier (IR-TF)}

Les expériences de spectroscopie infrarouge à transformée de Fourrier (IR-TF) ont été réalisées sur un spectromètre Perkin Elmer Spectrum 2000', en accumulant 20 scans de 4000 à $300 \mathrm{~cm}^{-1}$ avec une résolution de $2 \mathrm{~cm}^{-1}$ et un intervalle de $0,5 \mathrm{~cm}^{-1}$. Afin de minimiser la quantité d'échantillon utilisée, les analyses ont été faites en mode transmission en cellule diamant.

Les intensités relatives des bandes IR, déterminées grâce au logiciel OPUS ${ }^{\oplus}$, permettent de calculer trois rapports indicateurs de l'état de conservation du matériau (fig. 4) :

- le Splitting Factor $(S F)((\mathrm{a}+\mathrm{b}) / \mathrm{c}$ sur la figure 4) qui caractérise la cristallinité de la phase minérale de l'échantillon en se basant sur la séparation des bandes d'absorption IR $v_{4}$ $\mathrm{PO}_{4}^{3-}$ : plus le SF est grand, plus la cristallinité est élevée, plus la phase minérale est altérée $[10,11]$.

- La teneur en carbonate $\mathrm{CO}_{3}^{2-} / \mathrm{PO}_{4}^{3-}$ qui, par comparaison avec une référence moderne, permet d'évaluer les modification de la quantité de carbonates par rapport aux phosphates [10],[12].

La teneur en amide amide/PO ${ }_{4}^{3-}$ qui représente la quantité de phase organique présente, en prenant les phosphates comme référence [13].

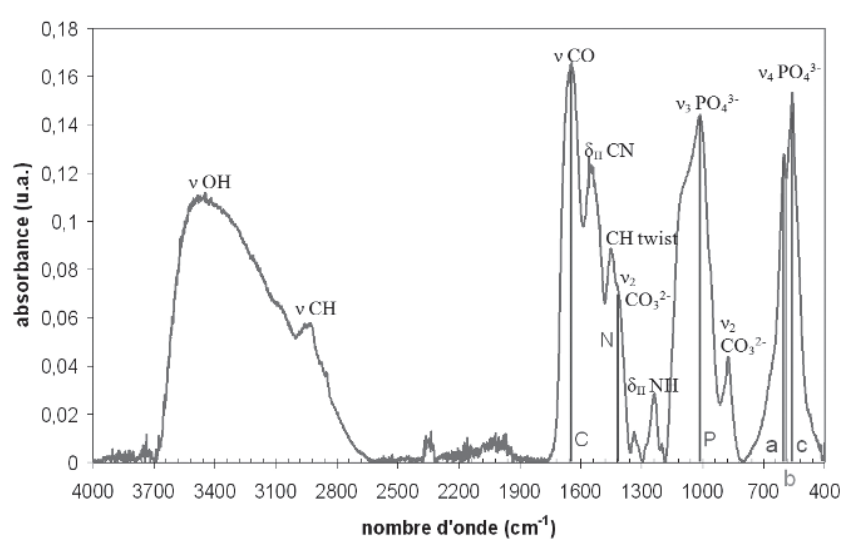

Figure 4: (Voir planche couleur) Bandes IR utilisées pour les calculs de coefficient, attribuées sur la base d'un spectre IR-TF d'ivoire moderne d'éléphant réalisé en mode transmission en cellule diamant.

Figure 4: (See colour plate) Identification of the IR bands used for the characteristic ratios, based on a FT-IR spectrum of modern elephant ivory obtained in transmission mode in a diamond cell.

\section{RÉsultats}

\section{Morphologie}

Dans la matrice compacte et homogène de l'ivoire moderne, de longues tubules de l'ordre d'un ou deux micromètres sont visibles sur les micrographies électroniques, leur orientation dépendant du sens de coupe de l'échantillon (fig. 5a). Pour les échantillons archéologiques de Jinsha, l'ivoire apparaît fractionné, principalement le long des joints de grains, mais des tubules sont visibles sous forme de longs rubans affleurants (fig. 5b), permettant de reconnaitre le matériau ivoire. La surface observée sur l'échantillon archéologique est accidentée. Cela est probablement dû à la grande fragilité du matériau, qui s'est effrité pendant ou après la préparation de l'échantillon par polissage.

Livoire archéologique montre aussi localement des zones de matrice qui paraissent plus claires sur les micrographies électroniques en mode BSE, donc plus denses en phase minérale, entourant des pores bien plus larges que ceux normalement observés dans l'ivoire (fig. 5c) : il s'agit des signes caractéristiques d'une attaque microbienne ou microbiologique [14].

L'image en trois dimensions des porosités obtenue par SR-microCT sur l'ivoire moderne montre des espaces vides continus et parallèles (fig. 6). Ce sont les tubules de la dentine dont on peut ainsi observer l'orientation dans l'échantillon. De plus, ces examens permettent une quantification de la porosité de 5,8 \% pour l'ivoire moderne (MI_El_1), de 

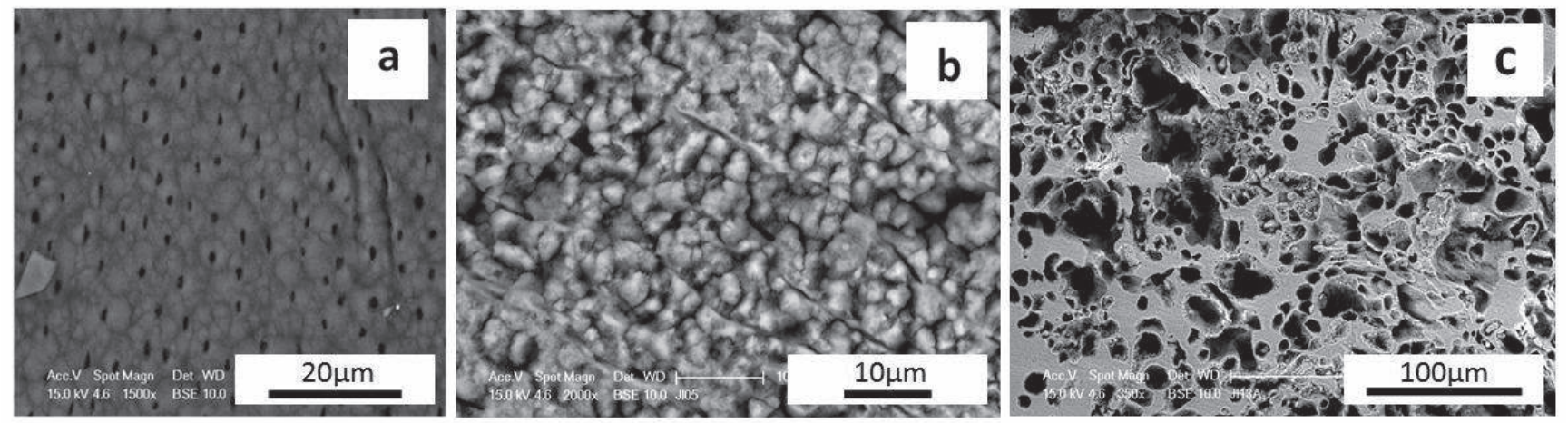

Figure 5 : Micrographie électronique en mode électrons rétrodiffusés : sur un échantillon moderne d'éléphant en coupe transverse MI_El_1 : a) tubules de l'ivoire, et sur l'échantillon archéologique AI_El_JI02b : b) tubules et c) faciès d'altération attribués à l'attaque microbiologique.

Figure 5: Electron micrographs in BSE mode: visible tubules a) on modern ivory sample ME_El_1 transversally cut, and b) on archaeological sample AI_El_JIO2b; c) characteristic features attributed to microbiological attack on archaeological sample AI_El_JIO2b.

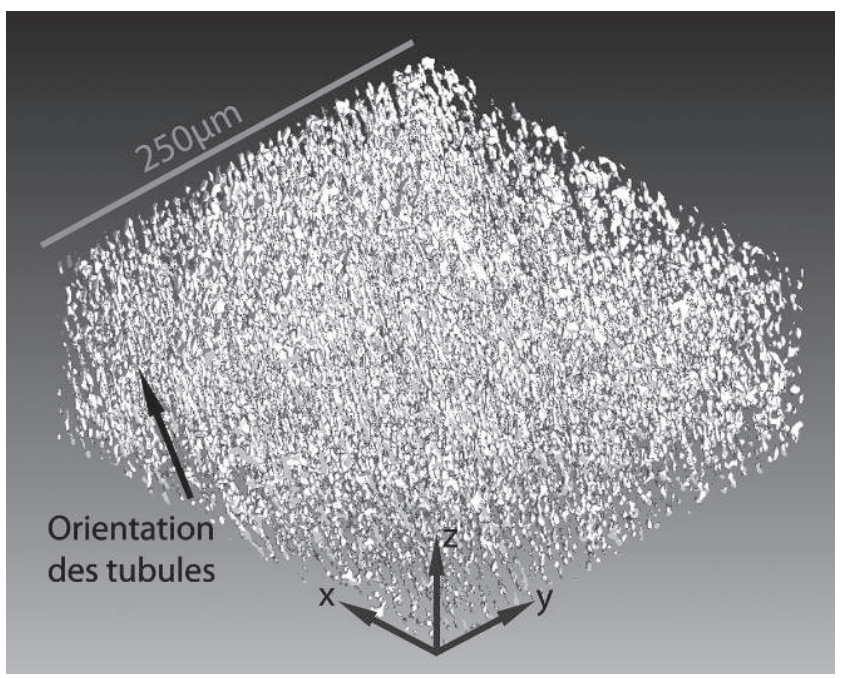

Figure 6 : Visualisation de l'orientation des tubules de l'ivoire moderne d'éléphant à partir de mesures de SR-microCT.

Figure 6: Representation of porosity and tubules orientation in modern ivory resulting from $S R$-microCT measurements.

28,1 \% pour le fragment archéologique AI_El_JI02b (celui où les traces d'altération biologique ont été observées) et de $2,5 \pm 1,1 \%$ pour l'échantillon AI_El_JI01d.

Les micrographies électroniques en transmission sur coupes ultrafines permettent d'observer directement la structure de l'ivoire à l'échelle nanométrique et en particulier les particules minérales dans la trame collagénique de la dentine. Deux types de cristaux semblent exister : certains, plus gros, sont distribués entre les fibres de collagène alors que d'autres, plus petits, se trouvent dans les fibrilles.
Cependant, l'arrangement observable des phases observé diffère grandement selon l'orientation de la coupe [7]. Ainsi, comme illustré par l'ivoire moderne, une coupe longitudinale (fig. 7a) permet d'observer la périodicité de l'organisation des cristaux entre les fibrilles de collagène (" banding " [15]) alors que les grands cristaux en forme d'aiguille sont bien visibles sur une coupe transverse (fig. 7b), et leur organisation laisse apercevoir les sections des fibrilles de collagène.

Pour les échantillons archéologiques, à cause de la petite taille et de l'altération des fragments, l'orientation de la coupe n'est hélas pas connue. Il est cependant évident que la disposition des cristaux est beaucoup plus aléatoire, bien que l'orientation préférentielle soit parfois encore discernable (fig. $7 \mathrm{~d}$ ). Les cristaux sont généralement plus gros que pour l'ivoire moderne et sont plus nombreux hors du plan de la coupe (fig. 7c) ce qui laisse penser qu'ils sont moins retenus par la trame organique que dans l'ivoire moderne. Cette observation ne semble pas être un artefact lié à la préparation de l'échantillon par ultramicrotomie mais de réels faciès.

\section{Composition des phases minérale et organique}

Les analyses microPIXE permettent de déterminer la composition élémentaire de l'ivoire en éléments majeurs, mineurs et traces à partir du sodium (tableau 3). Pour les échantillons archéologiques de Jinsha, et par rapport à la référence moderne, on note en particulier la perte relative du phosphore et du magnésium, ainsi que l'enrichissement relatif en calcium, silicium, fer et manganèse. De plus, les rapports $\mathrm{CaO} / \mathrm{MgO}$ pour les différents échantillons (fig. 8) permettent de différencier les trois défenses. 


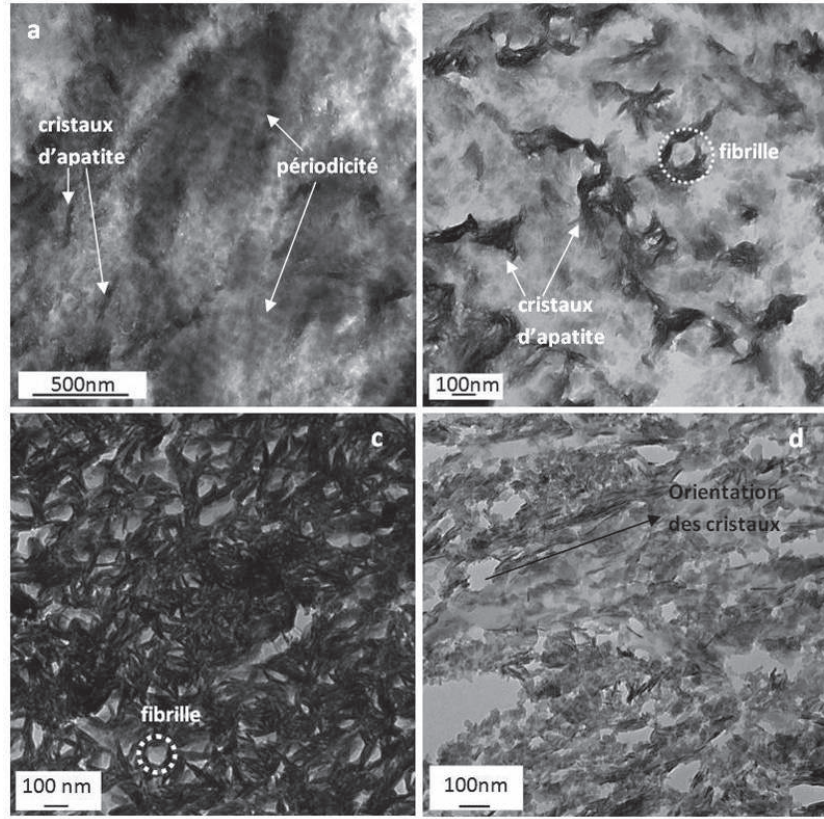

Figure 7 : Micrographies électroniques sur coupe ultrafine : de l'ivoire moderne MI_El_1 en coupe a) longitudinale et b) transverse, et des échantillons archéologiques c) AI_El_JI01d et d) AI_El_JI03g.

Figure 7: Electron micrographs obtained on ultrathin sections: modern ivory sample MI_El_1 cut along the longitudinal (a) and transverse (b) planes; archaeological samples $A I_{-} E l \_J I O 1 d$ (c) and $A I \_E l \_J I 03 g(d)$.

Les analyses en spectroscopie IR-TF permettent le calcul des coefficients SF, $\mathrm{CO}_{3}^{2-} / \mathrm{PO}_{4}^{3-}$ et amide/ $\mathrm{PO}_{4}^{3-}$ (tableau 4). L'augmentation de la cristallinité est notable pour les échantillons archéologiques, ainsi que l'appauvrissement en carbonates, particulièrement pour les défenses JI01 et JI03. De plus, les trois échantillons archéologiques montrent une perte d'environ $90 \%$ de leur phase organique par rapport à l'ivoire moderne.

\section{Discussion}

\section{Efficacité de la méthode adaptée à l'ivoire}

La démarche présentée ici a permis, par comparaison avec l'ivoire moderne, de caractériser l'altération de la phase minérale de la dentine, tant au niveau de sa composition élémentaire qu'au niveau de sa structure cristalline, et ce jusqu'à l'échelle du nanomètre. De plus, l'exploitation des spectres IR-TF permet d'estimer la quantité de collagène restant dans l'ivoire, paramètre primordial de sa conservation. Enfin, les observations optiques, électroniques et tomographiques nous informent sur la conservation de l'organisation hiérarchique du matériau et de l'imbrication de ses phases minérales et organiques. Ainsi les niveaux et composants essentiels de la dentine sont caractérisés.

\section{Comparaison entre l'ivoire d'éléphant moderne et prélèvements archéologiques}

Pour tous les prélèvements de Jinsha, nous remarquons d'abord la grande perte de phase organique par rapport à l'ivoire moderne. Les fibres de collagène étant le squelette de l'architecture hiérarchique de l'ivoire, il est généralement admis que leur désagrégation induise une perte d'organisation et de cohésion du matériau à toutes les échelles [12], ce qui explique en partie la grande fragilité et le délitement des échantillons visible au MEB, ainsi que la disposition aléatoire des cristaux observés au MET.

Pour la phase minérale, les variations de composition élémentaire par rapport à l'ivoire moderne sont d'autres indices d'altération car le transport d'espèces chimiques entre l'ivoire et le milieu d'enfouissement permet l'enrichissement en composés exogènes et/ou l'appauvrissent en composés constituants, faisant évoluer la composition du matériau. Pour les échantillons de Jinsha, la variation du rapport $\mathrm{CaO} / \mathrm{MgO}$ montre à la fois une diminution de la proportion relative en $\mathrm{Mg}^{2+}$ par rapport au phosphore et une

\begin{tabular}{|c|c|c|c|c|c|c|c|c|c|c|c|}
\hline Ivoire & $\mathrm{Na}_{2} \mathrm{O}$ & $\mathrm{MgO}$ & $\mathrm{Al}_{2} \mathrm{O}_{3}$ & $\mathrm{SiO}_{2}$ & $\mathrm{P}_{2} \mathrm{O}_{5}$ & $\mathrm{SO}_{3}$ & $\mathrm{Cl}$ & $\mathrm{CaO}$ & $\mathrm{MnO}^{2}$ & $\mathrm{TiO}_{2}$ & $\mathrm{Fe}_{2} \mathrm{O}_{3}$ \\
\hline MI_El_1 & 1,21 & 4,51 & 0,20 & - & 43,62 & 1,86 & 0,15 & 48,08 & - & 0,18 & 0,01 \\
\hline AI_El_JI01 & 0,44 & 0,49 & 0,19 & 1,34 & 40,13 & 0,69 & 0,12 & 53,78 & 0,26 & - & 2,35 \\
\hline AI_El_JI02 & 0,46 & 0,76 & 1,44 & 3,44 & 36,62 & 1,31 & 0,07 & 51,51 & 0,37 & - & 3,87 \\
\hline AI_El_JI03 & 0,52 & 0,29 & 0,26 & 0,50 & 40,01 & 0,64 & 0,10 & 55,73 & 0,15 & - & 1,53 \\
\hline
\end{tabular}

Tableau 3 : Compositions moyennes (\%) de la phase minérale obtenues par microPIXE pour l'ivoire moderne sur 23 analyses, et pour les sur échantillons archéologiques JI01, 02 et 03 sur 30, 4 et 18 analyses respectivement.

Table 3: Average composition (\%) of ivory mineral phase obtained by microPIXE on modern ivory (23 measurement points) and archaeological samples JIO1, 02 and 03 (30, 4 and 18 analyses, respectively). 


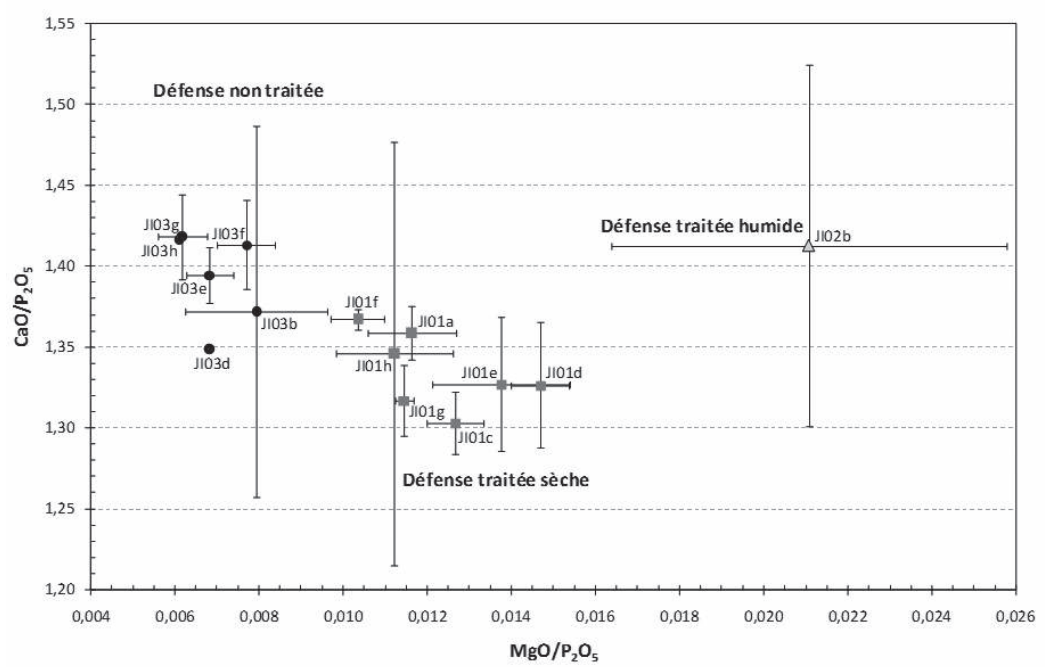

Figure 8 : Variation du rapport $\mathrm{CaO} / \mathrm{MgO}$ dans les prélèvements d'ivoire de Jinsha, déterminée par analyse microPIXE.

Figure 8: Variation of the $\mathrm{CaO} / \mathrm{MgO}$ ratios of the Jinsha ivory samples, obtained by microPIXE.

\begin{tabular}{|c|c|c|c|c|}
\hline Échantillon & Ivoire moderne & JI01e & JI02b & JI03f \\
\hline $\mathrm{SF}$ & $1.029 \pm 0,438$ & $2,830 \pm 0,798$ & $2,566 \pm 0,734$ & $3,313 \pm 0,266$ \\
\hline $\mathrm{CO}_{3}{ }^{2-} / \mathrm{PO}_{4}^{3-}$ & $0,375 \pm 0,089$ & $0,250 \pm 0,038$ & $0,354 \pm 0,076$ & $0,257 \pm 0,050$ \\
\hline amide/$/ \mathrm{PO}_{4}{ }^{3-}$ & $1,085 \pm 0,266$ & $0,097 \pm 0,020$ & $0,111 \pm 0,029$ & $0,073 \pm 0,013$ \\
\hline
\end{tabular}

Tableau 4 : Coefficients obtenus par spectroscopie IRTF, pour l'ivoire moderne, et les échantillons archéologiques JI01e, JI02b et JI03f, moyennés sur un minimum de six analyses.

Table 4: Parameters obtained by FT-IR spectroscopy on modern ivory and archaeological samples JIO1e, JIO2b and JIO3f (averages on at least six analyses).

augmentation de celle en $\mathrm{Ca}^{2+}$. Or, le magnésium, de par sa grande solubilité dans l'eau, est une espèce particulièrement sensible au lessivage [16-19], ce qui en fait un bon indicateur de l'état de conservation. Au vu du terrain très humide de Jinsha, nous pouvons donc penser que l'eau venant du sol d'enfouissement a engendré ce lessivage dans les échantillons archéologiques, provoquant la perte de magnésium et témoignant d'échanges importants entre l'ivoire et le milieu environnant.

À l'inverse de l'enrichissement en $\mathrm{Ca}^{2+}$ observé par microPIXE, classiquement lié à l'augmentation du taux de carbonates de calcium de l'échantillon, les résultats de spectroscopie IR-TF concluent en une teneur en carbonates plus faible dans les ivoires de Jinsha que dans l'ivoire moderne, impliquant a priori la dissolution d'une partie des carbonates de l'hydroxylapatite. L'augmentation de la teneur en $\mathrm{Ca}^{2+}$ pourrait être expliquée par la substitution au moins partielle des ions de $\mathrm{Mg}^{2+}$ par des ions $\mathrm{Ca}^{2+}$ venant du milieu d'enfouissement. Cependant cette hypothèse reste à être vérifiée.

Enfin, les coefficients SF des échantillons archéologiques sont tous plus élevés que celui de l'ivoire moderne, ce qui indique une cristallinité plus grande, confirmée par les modifications des cristaux observées au MET. Cette altération est certainement en partie due à l'activité des microorganismes, mais aussi à un mécanisme complexe de dissolution-recristallisation de l'hydroxylapatite qui intervient sur la phase minérale avec généralement pour effet une augmentation de la cristallinité.

\section{État de conservation des ivoires de Jinsha}

Wang et al. (2007) [20] ont déjà réalisé une première étude d'ivoires d'éléphant de Jinsha utilisant la spectroscopie IR-TF avec une référence moderne. Ils montrent une augmentation de la cristallinité de la phase minérale des échantillons archéologiques ainsi que l'introduction de carbonates dans la matrice apatique, concluant à leur recristallisation. Dans notre étude, la combinaison des coefficients $\mathrm{SF}$ et $\mathrm{CO}_{3}{ }^{2-1}$ $\mathrm{PO}_{4}^{3-}$ avec les mesures de $\mathrm{Ca}^{2+}$ par microPIXE confirme cette conclusion. Ne bénéficiant pas de datation fiable des défenses étudiées, nous n'avons cependant pas pu tester la corrélation annoncée entre l'âge de l'ivoire et la quantité d'ions $\mathrm{HO}^{-}$ et $\mathrm{CO}_{3}^{2-}$. De même, la répartition incertaine de nos prélèvements dans les défenses ne nous a pas permis d'observer d'évolution des bandes IR selon la position dans la défense. Toutefois, l'utilisation de méthodes analytiques complé- 
mentaires à l'IR-TF nous permet ici non seulement d'étayer les résultats d'évolution de la cristallinité par l'observation directe au MET des modifications de la taille des cristaux mais aussi de mieux visualiser l'état d'altération à l'échelle du micron de la dentine grâce aux micrographies électroniques obtenues au MEB. De plus, les analyses élémentaires décèlent d'autres phénomènes d'altération de la composition élémentaire et en particulier le lessivage du magnésium. Enfin, l'étude de Wang et al. [20] concluait à la perte totale de la phase organique et en faisait la première cause de la fragilité du matériau. Nos échantillons ne conservent plus que $10 \%$ environ de leur collagène d'origine et sont aussi relativement friables. Cette différence au niveau de l'état de conservation peut s'expliquer par des variations entre les défenses étudiées, d'âge ou de localisation par exemple. La conservation partielle de la matière organique est cependant en accord avec les analyses du sol donné par Wang et al. [20]. Un pH légèrement acide, de 6.5, et la présence d'argile sont généralement favorables à la conservation -au moins partielle- de la matière organique des matériaux osseux.

Nous avons montré que les phases organique et minérale des ivoires de Jinsha sont toutes deux altérées, probablement par des mécanismes impliquant l'eau de l'environnement humide d'enfouissement et l'activité des microorganismes. En effet, le mécanisme probable d'altération du collagène est ici l'hydrolyse par l'eau du milieu environnant, ce qui correspond au terrain très humide du site de Jinsha. De même, les échanges minéraux comme le lessivage du magnésium ou l'enrichissement en carbonates de calcium, très importants pour les échantillons de Jinsha, sont favorisés par un milieu humide, tout comme la dissolution-recristallisation de l'hydroxylapatite. Ainsi, l'altération de la dentine est un phénomène globale, qui atteint les phases organique et minérale, et ce à toutes les échelles.

\section{Différences d'état de conservation entre les défenses}

Il faut aussi noter les différences d'état de conservation entre les prélèvements de Jinsha et tout d'abord entre les défenses traitées (AI_El_JI01 et JI02) et celle non traitée (AI_El_JI03). En effet la teneur en phase organique des échantillons issus de cette dernière est $30 \%$ plus faible, ce qui indique une dégradation plus importante de la phase organique de la dentine. De plus, la cristallinité des échantillons non-traités est plus forte d'environ $20 \%$ que celle des défenses AI_El_JI01 et JI02, probablement à cause d'un phénomène de dissolution-recristallisation plus important ou de l'absence d'un biocide éventuel qui limiterait une attaque microbienne et empêcherait donc l'augmenta- tion de la porosité et de la cristallinité par cette activité. La phase minérale est aussi appauvrie de $45 \%$ en magnésium et enrichie de $6 \%$ en calcium en moyenne par rapport aux prélèvements traités, ces deux phénomènes dépendant d'échanges avec des éléments du milieu environnant. Ainsi, et selon tous les critères établis, l'ivoire de la défense non traitée est plus altéré que celui des défenses ayant bénéficié d'un traitement, bien que tous soient très fragiles. Il apparait donc que le traitement appliqué est partiellement efficace, a priori en limitant les échanges du matériau avec le milieu dans lesquels les microorganismes peuvent avoir un rôle.

De plus, entre les défenses traitées, l'une l'a été humide (AI_El_JI02) et l'autre sèche (AI_El_JI01). Les expériences de micro-tomographie ont montré une très grande différence de porosité entre les ivoires traités secs, moins poreux que l'ivoire moderne, et ceux traités humides, très poreux. La grande porosité de AI_El_JI02 peut être reliée aux traces d'attaques de microorganismes observées au MEB, dont une des conséquences est le creusement de cavités bien supérieures aux pores naturels. Cette plus grande porosité peut aussi être due à un autre état d'humidité entre la réféerence moderne et les échantillons archéologiques. En effet, l'ivoire a tendance à gonfler avec l'humidité ce qui peut se traduire par une augmentation de la taille des pores. Comme il parait peu probable que le traitement de surface ait entrainé la grande perte de porosité de l'ivoire traité sec, elle pourrait indiquer que de la matière externe à la matrice de la dentine aurait cristallisé dans les porosités de l'ivoires, comme les tubules, les comblant partiellement, ou que les pores se sont rétractés lors du processus de séchage. Cette hypothèse n'a pas pu être validée directement par observation au MEB, à cause de la petite taille et de la grande fragilité des échantillons. Ce phénomène n'ayant cependant $a$ priori pas eu lieu dans l'échantillon traité humide, nous pouvons en conclure qu'il a eu lieu pendant le séchage de l'ivoire et qu'il est entravé par le traitement, ce qui impliquerait les microorganismes dans la cristallisation de matériau exogène ou confirmerait la capacité du traitement à limiter les échanges avec le milieu. Cette dernière idée est renforcée par un appauvrissement moins important en magnésium ainsi qu'une teneur en carbonates de l'ivoire traité humide plus proche du matériau moderne, ses phénomènes dépendant aussi d'échanges avec l'extérieur.

De plus, les ivoires traités humides montrent une perte en phase organique légèrement plus faible que ceux traités secs. L'ensemble de ces résultats nous permet ainsi de conclure que, entre les défenses traitées, celle traitée sèche est plus altérée que celle traitée humide. Ainsi, le traitement est apparemment plus efficace appliqué sur le matériau encore humide. Comme au moment de la sortie de terre la majorité 
des échanges de l'ivoire avec le milieu environnant se fait pendant le séchage, cela s'articule logiquement avec l'effet présumé du traitement sur les échanges.

Cette comparaison entre défense traitée humide et traitée sèche confirme la forte influence du taux d'humidité et du séchage sur l'état de conservation de l'ivoire [2, 21]. Il apparaît ici que l'évaporation non contrôlée de l'eau gorgeant le matériau entraine probablement les espèces chimiques les plus solubles et favorise la recristallisation. La phase minérale fragilisée ne joue plus totalement son rôle protecteur vis-à-vis de la phase organique, permettant l'accélération de la dégradation du collagène. Il est donc possible que l'effet protecteur observé du traitement soit dû à une prolongation et une régulation, voulues ou non, de l'humidité du matériau, permettant un séchage moins rapide et agressif. Cependant, encore une fois, la nature inconnue du traitement limite le développement et la vérification de ces hypothèses, ainsi que l'évaluation de son efficacité. Nous pouvons toutefois conclure que pour les ivoires venant de milieux très humides, le séchage apparaît être le processus critique à surveiller et si possible à contrôler car il amplifie tous les phénomènes d'altération commencés dans le sol.

\section{Conclusion}

S'agissant d'un biomatériau nano-composite, des critères distinctifs de la macro- à la nano-échelle sont nécessaires pour décrire complètement l'ivoire et son état de conservation. De plus, pour estimer avec fiabilité son état de conservation, il faut prendre en compte à la fois sa phase minérale, sa phase organique ainsi que leur interaction. Nous avons montré dans cette étude qu'une méthodologie adaptée, pluriéchelle et multianalytique, permet de déterminer l'état de conservation des ivoires archéologiques à ces niveaux. Elle a tout d'abord été appliqué à l'ivoire moderne d'éléphant afin d'établir une base de référence pour les échantillons archéologiques. Ses performances ont ensuite été testées sur des échantillons du site archéologique de Jinsha présentant une forte fragilité. Cette méthodologie s'est montrée capable de quantifier l'altération de ses échantillons par rapport à la référence moderne ainsi que de différencier les échantillons entre eux.

Une distinction est de plus apparue entre les défenses traitées humides et celles traitées sèches, insistant une nouvelle fois sur l'importance de l'étape de séchage de l'ivoire à sa sortie de terre, en particulier pour des sites très humides comme celui de Jinsha.

Après le développement d'une approche analytique et des premières analyses de matériel moderne et archéologique, il est nécessaire de continuer à affiner la méthode et à élargir le panel des cas archéologiques étudiés. En particulier, il est important de mieux caractériser l'ivoire moderne, par ex. sur des coupes transverses ou longitudinales et d'approfondir nos connaissances sur ce matériau de référence à différentes échelles afin de comprendre ses hétérogénéités et ainsi de mieux pouvoir interpréter les résultats obtenus. L'application de cette méthode à de nouveaux corpus archéologiques caractéristiques d'autres conditions de conservation viendra ensuite compléter cette étude pour mieux comprendre les phénomènes primordiaux impliqués dans l'altération de l'ivoire.

\section{Remerciements}

Nous remercions Céline Chadefaux pour son aide pendant les premières analyses dans le cadre de sa thèse au C2RMF (20072009). Andreas Staude et Heinrich Riesemeier (BAM Berlin) sont remerciés pour l'acquisition et l'evaluation des données de microtomographie X au synchrotron BESSY II à Berlin. Danièle Jaillard (CCME Orsay) est remerciée pour son aide lors de la préparation des échantillons pour les observations au MET. Nous tenons aussi à remercier les collègues archéologues chinois du site de Jinsha et surtout le directeur Wang Yi pour nous avoir fait découvrir ce site et avoir mis à notre disposition les prélèvements d'ivoire. Enfin, l'ANR est remerciée pour son soutien financier grâce au programme de recherche ANR-07JCJC-0149 «ArBoCo».

\section{RÉFÉRENCES}

1. Locke, M., 2008 - Structure of Ivory. Journal of Morphology, 269, p. 423-450.

2. Godfrey, I. M. et al., 2002 - The analysis of ivory from marine environment. Studies in Conservation, 47, p. 29-45.

3. Archaeology, C.I.o.C.H.A., 2006 - ed. A $21^{\text {th }}$ Century Discovery of Chinese Archaeology. THE JINSHA SITE, Chinese Intercontinental Press.

4. Su, X. W. and CuI, F. Z., 1999 - Hierarchical structure of ivory: from nanometer to centimeter. Material Science and Engineering, C7, p. 19-29.

5. Dauphin, Y. and C. T. Williams, 2004 - Diagenetic trends of dental tissues. C. R. Palevol, 3, p. 583-590.

6. MülleR, K. and I. REICHE, 2011 - Differentiation of archaeological ivory and bone materials by micro-PIXE/PIGE with emphasis on two Upper Palaeolithic key sites: Abri Pataud and Isturitz, France. Journal of Archaeological Science 38(12), 3234-3243, DOI:10.1016/j.jas.2011.06.029. 
7. Jantou-Morris, V., Horton M. A. and McComb D. W., 2010

- The nano-morphological relationships between apatite crystals and collagen fibrils in ivory dentine, Biomaterials, 31(19), p. 5275-5286.

8. ReICHE, I. et al., 2011 - Synchrotron radiation and laboratory micro X-ray computed tomography-useful tools for the material's identification of prehistoric objects made of ivory, bone or antler. Journal of Analytical Atomic Spectroscopy26, 1802-1812, DOI:10.1039/C0JA00246A.

9. PICHON, L. et al., 2010 - A new mapping acquisition and processing system for simultaneous PIXE-RBS analysis with external beam. Nuclear Instruments and Methods in Physics Research B, 268, p. 2028-2033.

10. Collins, M. J. et al., 1998 - Deterioration of archaeological bone, European commission. Directorate general XII. Science, research and development. p. 1-65.

11. WeINER, S. and BAR-YoSEF O., 1990 - States of Preservation of Bones from Prehistoric Sites in the Near East: A Survey. Journal of Archaeological Science, 17, p. 187-196.

12. Wright, L. E. and Schwarcz, H. P., 1996 - Infrared and Isotopic Evidence for Diagenesis of Bone Apatite at Dos Pilas, Guatemala: Palaeodietary Implications. Journal of Archaeological Science, 23, p. 933-944.

13. REICHE, I. et al., 2010 - Microscale imaging of the preservation state of 5,000-year-old archaeological bones by synchrotron infrared microspectroscopy. Analytical and Bioanalytical Chemistry, 397, p. 2491-2499.

14. Müller, K. et al., 2011 - Microbial attack of archaeological bones versus high concentrations of heavy metals in the burial environment. A case study of animal bones from a medieval copper workshop in Paris. Palaeogeography, Palaeoclimatology, Palaeoecology, 310 (1-2), p. 39-51.

15. Jantou, V. et al., 2009 - Focused ion beam milling and ultramicrotomy of mineralised ivory dentine for analytical transmission electron microscopy. Micron, 40, p. 495-501.

16. Elliott, T. A. and Grime, G. W., 1993 - Examining the diagenetic alteration of human bone material from a range of archaeological burial sites using nuclear microprobe. Nuclear Instruments and Methods in Physics Research B, 77, p. 537 547.

17. HARE, P. E., 1980 - Organic geochemistry of bone and its relation to the survival of bone in the natural environment, in Fossils in the Making, Behrensmayer A. K. and Hill A. P., Editors, University of Chicago Press, Chicago, p. 208-219.

18. Parker, R. B. and H. Toots, 1980 - Trace elements in bones as palaeobiological indicators, in Fossils in the Making, A.K. Behrensmayer and A. P. Hill, Editors University of Chicago Press, Chicago.

19. Price, T. D., M. J. Schoeninger and G. Armelagos, 1985 - Bone Chemistry and Past Behavior: an Overview. Journal of Human Evolution, 14, p. 419-447.

20. WANG, L. et al., 2007 - Infrared spectroscopic study of modern and ancient ivory from sites at Jinsha and Sanxingdui, China Mineralogical Magazine, October, 71(5), p. 509-518.

21. Lafontaine, R. H. ANd P. A. Wood, 1982 - The stabilzation of ivory against relative humidity fluctuations. Studies in Conservation, 27, p. 109-117. 